\title{
Seizures Related to Antibiotic Use: Update
}

\author{
Celmir de Oliveira Vilaça*1,2, Marco Orsini ${ }^{3}$, Ricardo Martello ${ }^{3}$, Rossano Fiorelli ${ }^{3}$ and Cristiane Afonso ${ }^{4}$ \\ ${ }^{1}$ Department of Orthopedics, Institute of Traumatology and Orthopedics (INTO), Brazil
}

${ }^{2}$ Department of Neurology, Fluminense Federal University, Brazil

${ }^{3}$ Universidade Severino Sombra, Programa de Mestradoem Ciências Apliacadas à Saúde, Brazil

${ }^{4}$ Department of Epilepsy, Federal University of Rio de Janeiro, Brazil

Received: April 20, 2018; Published: May 04, 2018

*Corresponding author: Celmir Vilaça, Fluminense Federal University (UFF), Division of Neurology, Postgraduate Program in Neurology/NeurosciencesUFF, Niterói-RJ, Brazil

\begin{abstract}
Objective: make a review about the risk of seizures related to the use of antibiotics. Method: We perform a non-systematic review using Google Scholar platform approaching the relationship between seizures and the most used antibiotics classes in clinical practice. Results and Discussion: 34 papers in English language were used for this review.

Conclusion: Rarely antibiotics may cause seizures, mainly in patients with renal dysfunction, hepatic or previous brain disease. It is important to learn about drugs at greatest risk of seizures in each class of antibiotics. The treatment involves the use of correct medications, primarilygabaergic drugs, avoiding ineffective anticonvulsants.
\end{abstract}

Keywords: Seizures; Penicillins; Cephalosporins; Quinolones

\section{Introduction}

Epileptic seizures can be defined as the presence of involuntary muscle contractions of the whole body or part of it resulting from cerebral neuronal synchronous overshoot.It should not be regarded as an etiology in itself but as a syndrome of multiple origins [1,2]. Convulsive seizures are the immediate cause of hospitalization in up to 20 percent of patients in intensive care units (ICUs). As the initial cause of ICU admission, the main etiologies of seizures are mainly due to alcoholism or stroke [3]. Considering the entire period of hospitalization, convulsions occur in up to $10 \%$ of patients in general ICU and in 30 to $46 \%$ of patients in neurological units [4]. We can divide the etiology of the seizures into structural and metabolic with the aim of facilitating the therapeutic approach. Among the structural causes, in addition to stroke, we highlight the presence of primary and metastatic CNS tumors. Among the causes of metabolic origin in patients in the ICU, hyponatremia stands out [5].

In spite of being defined by the presence ofmuscular contractions, we emphasize the possibility of non-convulsive seizures. In these, there is loss of consciousness without the presence of involuntary movements, making diagnosis difficult.When prolonged; seizures may lead to non-convulsive status epilepticus (SE). The SE is set to last longer than 30 minutes or crisis occurrence of successive and recurrent seizures with incomplete recovery of consciousness between them [6]. The frequency of seizures or SE in intensive care units is very variable depending on the method used for its diagnosis, whether it is through the use of routine electroencephalogram (EEG) or continuous electroencephalographic monitoring [7]. On the other hand, we sometimes observe patients hospitalized in the ICU, where distant metabolic, structural and infectious causes seizures occur in close temporal relation to the beginning of some medication. It is estimated that $15 \%$ of drug-related seizures may manifest as status epilepticus. In this context, $10 \%$ of cases of status epilepticus in ICU patients may be due to the use of antibiotics. The high rate of seizures and the almost universal need for antibiotics during hospitalization in intensive care units make it essential to know the pathogenesisfrequency, risk factors and correct treatment of seizures related to antibiotics of the most used classes [8].

\section{Methods}

We did a non-systematic review of articles on the Google Scholar platform from January 1992 until February 2018.Preference was given to articles in Portuguese or English whose title presented the descriptors "seizures" or "epilepsy" associated with the most commonly used classes of antibiotics: penicillins, cephalosporins, quinolones, carbapenems, metronidazole and isoniazid. From this 
total we chose 34 articles in English to make this text. We include experimental articles, review articles, case reports, or expert opinions without any specific criteria for exclusion, unless written in English or Portuguese. Some papers were chosen from the references obtained from the articles of the initial search.

\section{Results and Discussion}

Most substances or toxins cause convulsive seizures through four distinct processes: the first mechanism is the imbalance between inhibitory and excitatory neurotransmitters. These include changes in the ratio of gamma-amino butyric acid (GABA)/ glutamate. The second derives from changes in the neuronal transmembrane ionic flux, especially the sodium and potassium ions. The third involves imbalance in the synthesis and release of biogenic amines such as dopamine, noradrenalin and acetylcholine [9]. The latter mechanism is related to conditions that alter the cerebral concentration of adenosine. Adenosine is a substance released by neurons in order to block excessive neuronal firing assisting in ending the crisis [10]. Since the 40s the epileptogenic potential of antibioticsis known from experiments involving the use of penicillins in the cortex of monkeys and intrathecal in humans.The first antibiotics related to the occurrence of seizures were penicillins, from the beta-lactam family $[11,12]$. Subsequently, penicillins and other classes of antibiotics were implicated in the occurrence of seizures. The risk of convulsions in the general population related to the use of antibiotics ranges from 0.04 to $0.5 \%$ depending on the class of antimicrobial used [13]. However, we emphasize that there are some patients, mainly patients in ICU under the use of polypharmacy.Thus, many other drugs can lower the seizure threshold, especially opioids and antidepressants. The latter correspond to both serotonin reuptake inhibitors and tricyclics with their anticholinergic effect [14].

\section{Penicillins}

Penicillins due to their antiquity are the most studied class of antibiotics and associated with the occurrence of seizures. They present epileptogenic potential associated with the antagonism of GABAA receptors by the $\beta$-lactam ring. This finging confirms the fact that ring removal eliminates the convulsive potential of this class of drugs [12]. GABA represents the major CNS inhibitory neurotransmitter being present in $30 \%$ of synapses. GABA receptors are divided into two subtypes: GABAA and GABA B. It is believed that GABAA antagonism is responsible for the pro-convulsive effect [15]. This antagonism occurs both by noncompetitive inhibition and by voltage-dependent changes [16]. In addition, synthetic penicillins are considered to have a lower risk of seizure when compared to natural penicillins (benzylpenicillins) [15]. Among the penicillins with a higher risk of seizures include crystalline penicillin (Penicillin G) [17]. The seizures related to antibiotic therapy with penicillin occur on average 12 to 72 hours after its onset and can begin up to two weeks later [15].

\section{Cephalosporins}

Cephalosporins, another group of drugs in the $\beta$-lactam family, also present GABAA receptor antagonism as a mechanism that triggers seizures.Its competitive inhibition of GABAA receptors when compared to non-competitive penicillins makes this class of antibiotics more epileptogenic [16]. We admit the epileptogenic potential of the cephalosporin to be associated with the size of the substituent at the 3 or 7 positions of the 7-cephalosporanic acid structure of its formation [14]. In addition, there is the possibility that cephalosporins contribute to crises through an agonist effect on glutamatergic N-methyl-D-Aspartate (NMDA) receptors [18]. Among drugs of this class most reports refer to cefazolin as the major cephalosporin associated with seizures [14]. Another drug also frequently described as pro-convulsant in this class is cefepime. Cefepime in this case presents a high incidence of nonconvulsive status $[19,20]$.

\section{Quinolones}

Very little is known about the mechanism of seizures involving quinolones when compared to $\beta$-lactams. The decrease in the convulsive threshold of quinolones can also be attributed to GABAergic antagonism and glutamatergic agonism [21]. In the case of GABA antagonism, quinolones act both by competitive inhibition of the receptor and by binding to benzodiazepine receptors in the GABA complex [16]. Among the quinolones at greatest risk for seizures, most cases mention ciprofloxacin [17]. On the other hand, there are studies evidencing that there is no increased risk of seizures with this class of antibiotics when administered orally. These cases would in fact represent an increased risk of seizures due to the etiology that led to the need for antibiotic therapy [22].

\section{Carbapenems}

This class of antimicrobial drugs is the most frequently associated with seizures in intensive care units. This is due to its wide spectrum of action, being frequently used in infections by multiresistant bacteria. Its pro-convulsive action is also related to gabaergic antagonism [23,24]. They also present a higher potential to decrease the seizure threshold compared to cephalosporins and penicillins because of their greater penetration into the CNS [17]. The difference in epileptogenic potential among drugs of this class may be related to differences in molecular structure, especially in the differences of the $\mathrm{C} 2$ side chain attached to the carbapenem ring.In this case the more hydrophobic side chain favors passage through the blood-brain barrier (BBB), increasing the risk of intoxication. Such fact would justify imipenem being the carbapenem most implicated in the occurrence of seizures [20]. Some studies suggest the possibility of imipenem seizures be associated with their joint application with cilastatin [14]. Another important factor in the use of this class of antibiotics is its interaction with valproic acid, decreasing its plasma levels. This basically occurs due to three reasons: In the first, carbapenems cause the entrapment of valproate within the erythrocytes, decreasing its serum level. Besides, carbapenems increase hepatic glucuronidation rates of valproate, thus also helping to lower blood levels. Finally, carbapenems may decrease the intestinal absorption of valproate [25].

\section{Isoniazid}

Isoniazid is a bacteriostatic antibiotic primarily used in the treatment of tuberculosis in our country and it presents a 
structure similar to pyridoxine. It is also the main drug implicated in suicide attempts in this group of patients [26]. Pyridoxine is a B-complex vitamin that acts as a cofactor for the enzyme glutamic acid-descaboxylase. This enzyme is responsible for the production of GABA from glutamic acid or glutamate. Thus, we can attribute the pro-convulsive effect of isoniazid to its antigabergic effect by pyridoxine antagonism [27]. In addition, without the action of glutamic acid decarboxylase, glutamate levels, the main excitatory transmitter of the brain, are increased, favoring excessive neuronal firing. Finally isoniazid binds to pyridoxine, forming inactive complexes eliminated by the kidneys, also contributing to the deficiency of pyridoxine [15]. The importance of the decrease in the reserves of pyridoxine and its relation with seizures can be verified by the existence of cases of epilepsy attributed to the lack of pyridoxine [28].

\section{Metronidazole}

Metronidazole is one of the most commonly used antibiotics in amoebic infections and anaerobic bacteria. The main adverse effect of metronidazole use is the occurrence of peripheral neuropathy [29]. Metronidazole is unique among antibiotics that cause encephalopathy or seizures. This is due to the fact that it is the only antibiotic associated with the presence of structural lesions in the central nervous system observed in routine radiological examinations, including tomography and cranial resonance $[23,30]$. In this case changes in structures of the cerebral midline are observed as in the region of the splenius of the corpus callosum and in the dentate nucleus of the cerebellum. These changes are mainly observed as hyperintense lesionsidentifiable in weighted MRI sequences in T2 [13,31]. The location of the lesions is similar to the lesions of midline structures found in the Wernicke-Korsakoff Encephalopathy. In addition to Wernicke's encephalopathy represent the main differential diagnosis of metronidazole encephalopathy, the similar location of the lesions suggests the interference of metronidazole in thiamine metabolism. In this case there is the formation of toxic radicals by the presence of metronidazole and its transformation into thiamine analogues $[29,30]$. Another mechanism by which metronidazole can lead to crisis would be through elevated levels of brain monoamines [32].

\section{Other Antibiotics}

There are rare reports of seizures associated with antibiotics of macrolides, aminoglycosides and oxazolidinonesclass, through unspecified mechanism. Despite its antiquity, good penetration in the CNS and frequent use in our environment, especially in patients with HIV, there is no report of convulsive crisis associated with the use of sulfamethoxazole with trimethoprim [17].

\section{Risk Factors Related to Patient For Antibiotic Seizures}

Among the factors responsible for the increased risk of seizures in patients under antibiotics, we can highlight renal failure, presence of liver disease, existence of epilepsy, previous brain disease and age. Renal failure, whether it be acute or chronic, increases the risk of seizures through multiple mechanisms. One of these would be the increase in the half-life of antibiotics in the circulation with greater risks of accumulations at doses considered toxic in the CNS. In addition, renal insufficiency causes proteinuria and hypoalbuminemia, with increase of free fraction not bound to plasma proteins of the antibiotics [23]. Finally, renal failure leads to the accumulation of anionic substancesof low molecular weight. These substances can saturate the mechanisms of active transport of CNS molecules into the blood through the BBB, making it difficult to remove the antibiotics from the cerebrospinal fluid [8].

The presence of liver disease as well as renal insufficiency may increase the plasma free fraction of certain antibiotics due to hypoalbuminemia by hepatic protein synthesis difficulty. There is also loss of cytochrome p450 function responsible for the metabolism of certain antibiotics [33]. The existence of cerebral disease or previous epilepsy may lead to a decrease in the convulsive threshold by neurotoxic antibiotics when compared to healthy individuals. This would be a result of increased permeability of the blood-brain barrier by preexisting brain disease $[8,16]$. Depending on the antibiotic class, the CSF of normal individuals may present 1 to $5 \%$ of the plasma antibiotic concentration. In cases of BBB change this concentration may increase to levels ranging from 20 to $30 \%$ [33]. Such fact also explains the increased risk of seizures when antibiotics are administered directly into the CNS during invasive procedures when compared to oral or intravenous administration [24].

On the other hand, it is precisely this increase in permeability in the underlying disease that causes the use of the antibiotic into effective doses for the treatment of infectious conditions. In these cases it may be difficult to differentiate crises from the underlying disease or the antibiotic [34]. In contrast, many diseases that cause convulsive threshold decrease are not primary of the CNS. We can mention the sepsis where the pyrogenic and inflammatory substances released can trigger convulsive events without a CNS etiology [33]. Age is another important risk factor for seizures caused by antibiotics. Senescence causes changes in absorption, metabolism of drugs by renal or hepatic alterations as well as changes in the ratio between free and protein-bound doses of the drugs. The accumulation of lipophilic drugs can also be altered by the greater amount of fat in the elderly when compared to muscle mass [20].

\section{Diagnosis}

We should suspect antibiotic-related seizures in every patient under the use of it with no other cause specific for seizures. In ICU the difficulty of recovery of consciousness even in the presence of improvement of infectious parameters such as fever and leucometria after withdrawal of sedation increases the possibility of the presence of non-convulsive status epilepticus. Focal or secondarily generalized seizures increase the need for investigation through imaging for the presence of structural causes.8EEG is essential in the diagnosis of ES, although unspecific findings are often observed. These are characterized by slower latencies, of great amplitude, as well as generalized periodic discharges with three-phase morphology [23]. Lastly, although it is possible to dose antibiotic levels, there is no defined toxic level for most of these drugs. Besides, individual variability, immediate need for 
treatment, and waiting time for the test result make the dosage of currently measured antibiotic levels unjustified [33].

\section{Treatment}

Once the seizure disorder attributed to the use of antibiotics has been diagnosed, the antibiotic exchange for another of the same spectrum with less epileptogenic capacityis essential. The fact that most antibiotics act through gonagic antagonism indicates the preferencefor GABA agonist drug use. The use of benzo diazepines and barbiturates is particularly note worthy $[5,18]$. Thus, we emphasize the in efficacy of phenytoin and carbamazepine use due to the absence of GABA receptor activity. These drugs work mainly on sodium channels [24]. In case of carbapenem use it is essential to avoid using or increasing the administered dose of valproic acid if it is already in use. As an alternative to theincreasing dose of valproate, the use of adjuvant drug during carbapenem usemay is indicated. In this case, the drug should be maintained on average up to two weeks after the carbapenem has been discontinued, and benzodiazepine is generally used [25]. In cases of seizures related to isoniazid, the lack of pyridoxine to produce GABA makes useless not only the use of drugs with no effect on GABAA receptors but also the inefficacy of gabaergic drugs, with indication of pyridoxine replacement [27]. In the event of crises caused by metronidazole in addition to its suspension, thiamine replacement is indicated. However, due to the presence of structural lesions in metronidazole intoxication, there is an important risk of sequelae [31]. It is rarely possible to withdraw some antibiotics through dialysis, especially $\beta$-lactams, and the risk of recurrence of seizures is unknown with the exchange of the causative antibiotic by another antimicrobial of the same class [13].

\section{Conclusion}

Despite the rarity, it is important to pay attention to the possibility of seizures attributed to antibiotic therapy, especially in patients in intensive care units. It increases the suspicion of seizures by antibiotics in the presence of renal, hepatic or previous CNS disease in case of improvement of the infectious condition and the EEG should be requested.In addition to the knowledge about major seizure-related antimicrobials, the correct choice of medication for treatment is essential. Preference should be given to gabaergic drugs, avoiding carbamazepine or phenytoin as well as harmful interactions of antibiotics with prescribed anticonvulsants prior to hospitalization.

\section{References}

1. Lafrance WC (2010) Issues In Psychogenic nonepileptic seizures or attacks ? It' s not just semantics : Seizures.Neurology 75(1): 87-88.

2. Fisher RS,Emde Boas W Van, Blume W, Elger C, Genton P, et al. (2005) Epileptic seizures and epilepsy: Definitions proposed by the International League Against Epilepsy (ILAE) and the International Bureau for Epilepsy (IBE). Epilepsia 46(4): 470-472.

3. Vyskocilova D, Klimosova S (2010) Epileptic seizures as a primary cause for admission to neurology intensive care unit. Neurolasia15(1): 42.

4. Schmitt SE (2017) Utility of Clinical Features for the Diagnosis of Seizures in the Intensive Care Unit. J Clinical Neurophysiology 34(2): 158-161.
5. Varelas PN, Mirski M (2007) Treatment of seizures in the neurologic intensive care unit. Curr Treat Options Neurol 9(2): 136-145.

6. Fernandez Torre JL, Martinez Martinez M, GonzalezRato J, Maestro I, Alonso I, et al. (2005) Cephalosporin-induced Nonconvulsive Status Epilepticus: Clinical and Electroencephalographic Features. Epilepsia 46(9): 1550-1552.

7. Varelas PN, Spanaki MV, Mirski MA (2013) Seizures and the Neurosurgical Intensive Care Unit. Neurosurg Clin N Am 24(3): 393-406.

8. Misra UK, Kalita J, Chandra S, Nair PP (2013) Association of antibiotics with status epilepticus. NeurolSci34(3): 327-331.

9. Sharma AN, Hoffman RJ (2011) Toxin-Related Seizures. Emerg Med Clin North Am29(1): 25-139.

10. Boison D (2005) Adenosine and Epilepsy: From Therapeutic Rationale to New Therapeutic Strategies. Neuroscientist 11(1): 25-36.

11. Keskin S, Konkol RJ (1993) Seizure Exacerbation Related to Betalactam Antibiotics in a Child with Cerebral Dysgenesis. Dev Med Child Neurol 35(3): 267-274.

12. Grøndahl TO, Langmoen IA (1993) Epileptogenic effect of antibiotic drugs. J Neurosurg 78(6): 938-943.

13. Bhattacharyya S, Darby R, Berkowitz AL (2014) Antibiotic-Induced Neurotoxicity. Curr Infect Dis Rep 16(12): 448.

14.Arkaravichien W, Tamungklang J, Arkaravichien T (2006) Cefazolin induced seizures in hemodialysis patients. J Med Assoc Thai89(11): 1981-1983.

15. Wallace KL (1997) Antibiotic-Induced Convulsions. Crit Care Clin 13(4): 741-762.

16. Esposito S,Canevini MP, PrincipiN (2017) Complications associated with antibiotic administration: neurological adverse events and interference with antiepileptic drugs. Int J Antimicrob Agents 50(1): 1-8.

17. Sutter R, Rüegg S, Tschudin Sutter S (2015) Seizures as adverse events of antibiotic drug : A systematic review. Neurology 85(15): 1332-1341.

18. Sugimoto M, Uchida I, Mashimo T, Yamazaki S, Hatano K, et al. (2003) Evidence for the involvement of $\operatorname{GABA}(\mathrm{A})$ receptor blockade in convulsions induced by cephalosporins. Neuropharmacology 45(3): 304-314.

19. Whitney C, Worthington J, Gudur UM, Nookala V(2017)Cefepime Induced Neurotoxicity in an ICU Patient 3: 3-4.

20. Mattappalil A, Mergenhagen KA (2014) Neurotoxicity with Antimicrobials in the Elderly: A Review. ClinTher36(11): 1489-1511.

21. Bellon A, PerezGarcia G, Coverdale JH, Chacko RC (2009) Seizures associated with levofloxacin: Case presentation and literature review. Eur J Clin Pharmacol 65(10): 959-962.

22. Chui CSL, Chan EW, Wong AYS, Root A, Douglas IJ, et al. (2016) Association between oral fluoroquinolones and seizures: A self-controlled case series study. Neurology 86(18): 1708-1715.

23. Bhattacharyya S, Darby RR, Raibagkar P, Gonzalez Castro LN, Berkowitz AL (2016) Antibiotic-associated encephalopathy. Neurology 86(10): 963-971.

24. Yamazaki S, Mochizuki Y, Terai T, Sugimoto M, Uchida I, et al. (2002) Intracerebroventricular injection of the antibiotic cefoselis produces convulsion in mice via inhibition of GABA receptors. Pharmacol Biochem Behav 74(1): 53-59.

25. Huang CR, Lin CH, Hsiao SC, Chen NC, Tsai WC, et al. (2017) Drug interaction between valproic acid and carbapenems in patients with epileptic seizures. Kaohsiung J Med Sci 33(3):130-136.

26. Tsubouchi K, Ikematsu Y, Hashisako M, Harada E, Miyagi H, et al. (2014) Convulsive Seizures with a Therapeutic Dose of Isoniazid. Intern Med 53: 239-242. 
27. Glatstein M, Carbell G, Scolnik D, Rimon A, Banerji S, et al. (2018) Pyridoxine for the treatment of isoniazid-induced seizures in intentional ingestions: The experience of a national poison center. Am J Emerg Med 735-6757(18) 30100-301001.

28. GülMert G, İncecik F, Hergüner MÖ, Ceylaner S, Altunbaşak Ş (2015) Pyridoxine-dependent epilepsy in two Turkish patients in Turkey and review of the literature. Turk J Pediatr 57(4): 394-397.

29. Chen LA, Huang YT, Cheng SJ (2012) Metronidazole-induced Encephalopathy: Case Report and Review Literature. Acta Neurol Taiwan 21(2): 74-78.

30. LyD, DeLisi LE (2017) Can antibiotics cause a psychosis? Case report and review of the literature. Schizophr Res 189: 204-207.

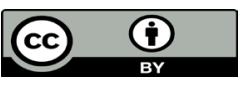

This work is licensed under Creative Commons Attribution 4.0 License

Submission Link: https://biomedres.us/submit-manuscript.php
31. Hobbs K, SternNezer S, Buckwalter MS, Fischbein N, Finley Caulfield A (2015) Metronidazole-Induced Encephalopathy: Not Always a Reversible Situation. Neurocrit Care 22(3): 429-436.

32. Godthaab Sørensen C, Karlsson WK, Amin FM, Lindelof M (2018) Convulsive Seizures as Presenting Symptom of Metronidazole-Induced Encephalopathy: A Case Report. Case Rep Neurol 10(1): 34-37.

33. Deshayes S, Coquerel A, Verdon R (2017) Neurological Adverse Effects Attributable to $\beta$-Lactam Antibiotics: A Literature Review. Drug Saf 40(12): 1171-1198.

34. Haldar R, Kaushal A, Gupta D, Srivastava S, Singh P, et al. (2015) Convulsions following rapid ceftazidime administration for preinduction antibiotic prophylaxis during neurosurgical procedure. Anesth Essays Res 9(3): 427-429.

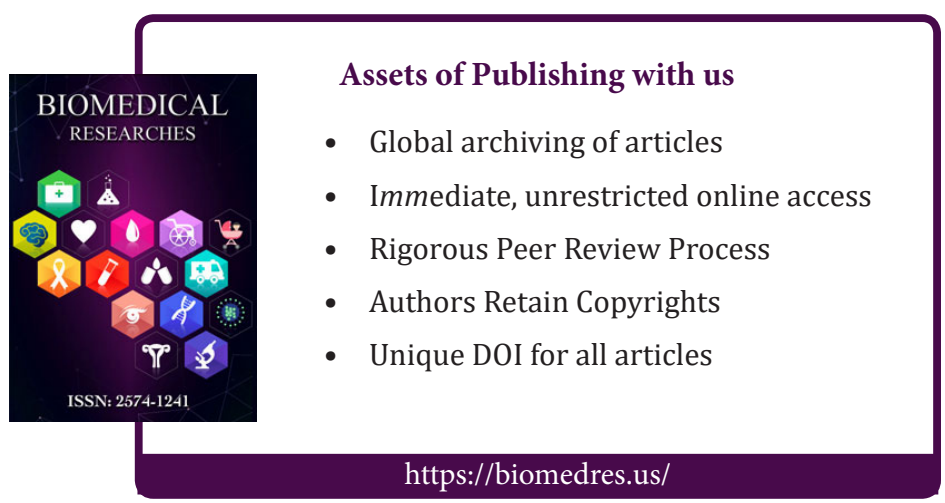

